\title{
Comparison of two-drug combinations, amikacin/tigecycline/ imipenem and amikacin/tigecycline/clarithromycin against Mycobacteroides abscessus subsp. abscessus using the in vitro time-kill assay
}

\author{
Elena Portell-Buj ${ }^{1} \cdot$ Queralt Bonet-Rossinyol $^{2,3} \cdot$ Alexandre López-Gavín $^{2} \cdot$ Angely Roman $^{2}$. \\ Mariana Fernández-Pittol ${ }^{2} \cdot$ Griselda Tudó $^{1} \cdot$ Julian Gonzalez-Martin ${ }^{2}$
}

Received: 4 August 2020 / Revised: 26 October 2020 / Accepted: 9 November 2020 / Published online: 8 January 2021

(c) The Author(s) 2021. This article is published with open access

\begin{abstract}
Nontuberculous mycobacteria include 198 mycobacterial species. Among these, Mycobacteroides abscessus is a rapidly growing mycobacteria that causes lung and skin infections. M. abscessus lung infections are difficult to treat due to the high levels of resistance to several classes of antibiotics. The current treatment is based on combining at least two or three antibiotics. However, treatment outcomes remain very poor. The objective was to compare the in vitro activity of amikacin, tigecycline, imipenem, and clarithromycin, alone and in two different three-drug combinations (amikacin/tigecycline/imipenem and amikacin/tigecycline/ clarithromycin) against seven M. abscessus subsp. abscessus clinical isolates using the time-kill assay. The two combinations showed greater activity than the antibiotics tested individually. Even though both combinations showed similar activity as well as no antagonistic activity, the combination including imipenem could not be an alternative treatment against $M$. abscessus subsp. abscessus lung infections caused by clarithromycin susceptible isolates. However, this combination could be considered against clarithromycin resistant isolates. Future studies are necessary to confirm this hypothesis.
\end{abstract}

\section{Introduction}

The nontuberculous mycobacteria (NTM) family includes 198 species, the majority of which do not cause disease in humans [1]. NTM are naturally found in the environment,

These authors contributed equally: Griselda Tudó, Julian GonzalezMartin

\section{Julian Gonzalez-Martin}

gonzalez@clinic.cat

1 Departament de Fonaments Clínics, Facultat de Medicina i Ciències de la Salut, Universitat de Barcelona, Servei de Microbiologia, CDB, Hospital Clínic de Barcelona-ISGlobal, c/ Villarroel 170, 08036 Barcelona, Spain

2 Servei de Microbiologia, CDB, Hospital Clínic de BarcelonaISGlobal, Departament de Fonaments Clínics, Facultat de Medicina i Ciències de la Salut, Universitat de Barcelona, c/ Villarroel 170, 08036 Barcelona, Spain

3 Laboratory of Molecular Microbiology, Biology Department, University of Girona, Girona, Spain from soil to water systems [2]. Recently, mycobacterial species have been divided into five new genera: Мycobacterium, Mycolicibacter, Mycolicibacterium, Mycolicibacillus, and Mycobacteroides [3]. Over the last years, infections caused by NTM have significantly increased [4]. Among these NTM, Mycobacteroides abscessus is a rapidly growing mycobacteria (RGM) that causes lung and skin infections which are difficult to treat due to its resistance to most classes of antibiotics [2, 4]. This RGM is classified into three subspecies: M. abscessus subsp. abscessus, $M$. abscessus subsp. massiliense and M. abscessus subsp. bolletii $[2,3,5]$. The identification to subspecies level is important since their resistance profiles to macrolides are different. Macrolides, and specifically clarithromycin are among the most active antibiotics against $M$. abscessus. The resistance to macrolides can be either constitutive ( $r r l$ gene) or inducible (erm41 gene). The most frequent is the inducible resistance, due to the presence of a functional erm 41 gene that codes for a methylase. Accordingly, M. abscessus subsp. massiliense is usually susceptible to macrolides since the erm 41 gene is truncated and not functional. $M$. abscessus subsp. bolletii is innately resistant to macrolides. 
Most of M. abscessus subsp. abscessus also exhibit inducible macrolide resistance, but some isolates remain susceptible due to a non-functional erm 41 gene. Although there are geographical differences, the most commonly isolated is M. abscessus subsp. abscessus [4, 6].

M. abscessus lung infections are particularly common in patients with underlying respiratory conditions, such as cystic fibrosis and bronchiectasis [5]. These infections require treatment, but management is difficult due to high levels of natural and acquired resistance to frequently used antibiotics. Moreover, the presence of mucus and other secretions at the site of infection makes the entry of antibiotics difficult. Depending on the macrolide resistance profile, the current guidelines of the British Thoracic Society recommend the administration of different intravenous (IV) and oral antibiotics (e.g., IV amikacin, IV tigecycline, IV imipenem, and oral clarithromycin) for 1 month during the initial phase followed by oral and inhaled antibiotics (e.g., oral clofazimine, oral moxifloxacin and nebulized amikacin) for a minimum of 12 months during the continuation phase. The use of macrolides is recommended even in the presence of inducible resistance, but not when constitutive resistance associated to the $r r l$ gene is found $[6,7]$. On the other side and despite its IV administration, tigecycline has better activity than former drugs used in combination with amikacin, such as doxycycline, tetracycline and minocycline [8]. Nevertheless, side effects are common and treatment outcomes are very often poor [2]. Hence, it is of great importance to develop new antibiotics and antibiotic combinations.

The treatment regimens available for M. abscessus lung infections consist of the administration of different antibiotics in combination. Therefore, it is necessary to determine the efficacy of these combinations as well as of that of the antibiotics alone. The time-kill assay establishes the in vitro pharmacodynamics of antibiotics by detecting the rate at which an antibiotic concentration kills bacteria along time [9].The main goal was to compare the in vitro activity of four different antibiotics, amikacin, tigecycline, imipenem, and clarithromycin alone and in two different three-drug combinations (amikacin/tigecycline/imipenem and amikacin/tigecycline/clarithromycin) against $M$. abscessus subsp abscessus clinical isolates using the in vitro time-kill assay.

\section{Material and methods}

\section{Mycobacteroides abscessus subsp. abscessus clinical isolates}

Seven clinical isolates of M. abscessus subsp. abscessus were selected from a strain collection of the Microbiology
Department of the Hospital Clinic of Barcelona (Spain). They were previously identified at subspecies level using GenoType NTM-DR (Hain Lifescience GmbH, Nehren, Germany). In addition, Sensititre ${ }^{\mathrm{TM}}$ AST RAPMYCO plates (Thermo Fisher Scientific, MA, USA) were used in accordance to manufacturers recommendations for antibiotic susceptibility testing, incubating the plates for 3 days, except for clarithromycin whose incubation was prolonged for up to 7 and 14 days to detect the presence of inducible resistance. The seven isolates selected had the following range of minimum inhibitory concentrations (MICs) to the antibiotics tested: $4-16 \mu \mathrm{g} \mathrm{ml}^{-1}$ for amikacin, $0.125-0.5 \mu \mathrm{g} \mathrm{ml}^{-1}$ for tigecycline, $8-64 \mu \mathrm{g} \mathrm{ml}^{-1}$ for imipenem, and $0.5-4 \mu \mathrm{g} \mathrm{ml}^{-1}$ for clarithromycin (Table 1). Stocks of each isolate were preserved at $-80^{\circ} \mathrm{C}$ in skim milk and were thawed for each assay.

\section{Antibiotics}

The four antibiotics tested were purchased from SigmaAldrich (St. Louis, MO, USA). Amikacin and imipenem were dissolved in sterile distilled water. Clarithromycin was dissolved in dimethyl sulfoxide (DMSO) $(0.002 \%$ final concentration) and sterile distilled water. All the antibiotics were sterilized by filtration and stored at $-20^{\circ} \mathrm{C}$.

\section{Tigecycline preparation}

Tigecycline was prepared as described by Jitkova et al. [10] due to its poor stability. It was dissolved in DMSO $\left(1 \mathrm{mg} \mathrm{ml}^{-1}\right)$ and saline solution containing ascorbic acid $\left(3 \mathrm{mg} \mathrm{m}^{-1}\right)$ (Sigma-Aldrich) and pyruvate $\left(60 \mathrm{mg} \mathrm{ml}^{-1}\right)$ (Sigma-Aldrich) and adjusted to $\mathrm{pH}$ 7.0. With this formulation, tigecycline remained stable for up to 7 days when protected from light [10].

Table 1 Minimum inhibitory concentrations (MICs) of the $M$. abscessus clinical isolates studied

\begin{tabular}{lclcl}
\hline Isolate & \multicolumn{2}{l}{ MICs $\left(\mu \mathrm{g} \mathrm{ml}^{-1}\right)$} & \\
\cline { 2 - 4 } & $\mathrm{AMK}$ & $\mathrm{TGC}$ & $\mathrm{IPM}$ & CLR \\
\hline M. abscessus 1 & 8 & 0.125 & 64 & 0.5 \\
M. abscessus 2 & 8 & 0.5 & 16 & 4 \\
M. abscessus 3 & 16 & 0.25 & 8 & 1 \\
M. abscessus 4 & 16 & 0.25 & 64 & 0.5 \\
M. abscessus 5 & 8 & 0.25 & 8 & 2 \\
M. abscessus 6 & 8 & 0.25 & 32 & 0.5 \\
M. abscessus 7 & 4 & 0.5 & 8 & 0.5 \\
M. abscessus MIC $_{90}$ & 16 & 0.5 & 64 & 4 \\
\hline
\end{tabular}

AMK amikacin, $T G C$ tigecycline, IPM imipenem, $C L R$ clarithromycin 


\section{Inoculum preparation}

All of the isolates were grown in $\mathrm{BD}^{\mathrm{TM}}$ Columbia Agar with 5\% Sheep Blood plates (Becton Dickinson, Sparks, MD). Then, they were subcultured in Middlebrook $7 \mathrm{H} 9$ liquid medium (Becton Dickinson) supplemented with 10\% oleic acid-albumin-dextrose-catalase (Comercial Bellés, Tarragona, Spain) and 0.25\% Tween 80 (Merck, Darmstadt, Germany) to avoid bacilli clump formation. Finally, the $M$. abscessus subsp abscessus cultures were homogenized by agitation and adjusted to the desired concentration using a nephelometer (CrystalSpec ${ }^{\mathrm{TM}}$; Becton Dickinson).

\section{Minimum inhibitory concentrations}

The MICs of each antibiotic were determined in 96-well plates (Smartech Biosciences, Barcelona, Spain). Briefly, $100 \mu \mathrm{l}$ of Mueller Hinton broth were added to each well. Then, serial dilutions of the antibiotics ranging from 512 to $0.25 \mu \mathrm{g} \mathrm{ml}^{-1}$ were made. Finally, $100 \mu \mathrm{l}$ of inoculum at a concentration of $0.5 \mathrm{McFarland}\left(5 \times 10^{6} \mathrm{CFU} \mathrm{m}{ }^{-1}\right)$ were added. Positive control wells consisted of $100 \mu$ of Mueller Hinton and $100 \mu \mathrm{l}$ of inoculum $\left(5 \times 10^{6} \mathrm{CFU} \mathrm{ml}{ }^{-1}\right)$. Negative control wells were prepared by adding $200 \mu \mathrm{l}$ of Mueller Hinton. Plates were incubated at $30^{\circ} \mathrm{C}$ for 3 days and for up to 14 days in the case of clarithromycin. After incubation, visual reading was performed. The MIC was interpreted as the lowest antibiotic concentration preventing growth. All the experiments were performed in duplicate.

\section{Time-kill assays}

A previously described protocol was adapted for the present study [11]. Briefly, the time-kill assays were performed by dispensing $600 \mu \mathrm{l}$ of the corresponding antibiotic concentration (amikacin, tigecycline, imipenem, or clarithromycin) and $500 \mu \mathrm{l}$ of inoculum (final concentration of $5 \times 10^{5} \mathrm{CFU} \mathrm{ml}^{-1}$ ) into tubes with $7.8 \mathrm{ml}$ of Mueller Hinton broth. For the three-drug combinations (amikacin/tigecycline/imipenem and amikacin/tigecycline/clarithromycin), $200 \mu \mathrm{l}$ of each of the three antibiotics were dispensed into the tubes. Amikacin and imipenem were tested at $2 \times$ MIC and tigecycline and clarithromycin at $4 \times$ MIC. Control growth tubes containing $500 \mu \mathrm{l}$ of sterile distilled water instead of antibiotic were also included. All of the tubes were incubated at $37^{\circ} \mathrm{C}$ in a $5 \% \mathrm{CO}_{2}$ atmosphere for 6 days. Time points were established at days $0,1,3$, and 6 . At the defined time points, a volume of $500 \mu \mathrm{l}$ was removed from each liquid culture. The number of viable mycobacteria in each culture was determined by plating 10 -fold serial dilutions on Middlebrook 7H11 medium (Becton Dickinson). The $7 \mathrm{H} 11$ agar plates were incubated at $37{ }^{\circ} \mathrm{C}$ in a $5 \% \mathrm{CO}_{2}$ atmosphere for 3 days, after which colony-forming units were counted. All the experiments were performed in duplicate.

\section{Data analysis of the time-kill assays}

The means of the $\log _{10} \mathrm{CFU} \mathrm{ml}{ }^{-1}$ values were plotted against time for each isolate. The results were interpreted by the effect of the combinations compared with the most active antibiotic tested individually. Synergy was considered when the activity of the combinations was $2 \log _{10}$ higher than the most active antibiotic alone. Antagonism was determined when the activity of the combinations was

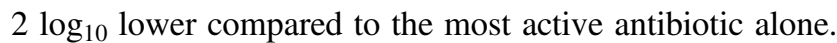
Finally, a difference of less than $2 \log _{10}$ lower or higher was considered indifferent.

\section{Statistical analysis}

Significant differences between the two combinations (amikacin/tigecycline/imipenem or amikacin/tigecycline/ clarithromycin) were analyzed using the Wilcoxon Mann-Whitney test. Results showing $P \leq 0.05$ were considered as statistically significant. Calculations were performed using STATA 13.0 software (Stata Corporation, College Station, TX, USA).

\section{Results}

Four different antibiotics, amikacin, tigecycline, imipenem, and clarithromycin, alone and in combination (amikacin/tigecycline/imipenem and amikacin/tigecycline/clarithromycin) were tested against clinical isolates of M. abscessus subsp. abscessus using the time-kill assay. The results observed during the 6-day period are summarized in Table 2.

In the present study at day 6, amikacin and tigecycline showed a mean 2.01 and $0.46 \log _{10} \mathrm{CFU} \mathrm{m}{ }^{-1}$ decrease, respectively, compared with the initial inoculum (5.70 $\log _{10}$ $\mathrm{CFU} \mathrm{m}{ }^{-1}$ ). Imipenem and clarithromycin displayed a mean 1.48 and $1.27 \log _{10} \mathrm{CFU} \mathrm{ml}{ }^{-1}$ decrease, respectively. The combination of amikacin/tigecycline/imipenem showed a mean decrease of $2.58 \log _{10} \mathrm{CFU} \mathrm{ml}^{-1}$. Finally, the combination of amikacin/tigecycline/clarithromycin displayed a mean decrease of $3.02 \log _{10} \mathrm{CFU} \mathrm{ml}^{-1}$. Although being less pronounced, these decreases were also observed at day 3 .

No significant differences were observed among the two replicates of each isolate. The most active individual antibiotic was amikacin followed by imipenem and clarithromycin. Both combinations were indifferent (neither synergistic nor antagonistic) showing more activity than the antibiotics tested individually. Moreover, no significant 
Table 2 Mean colony-forming values $\left(\log _{10} \mathrm{CFU} \mathrm{ml}{ }^{-1}\right)$ and reduction in growth compared with the initial inoculum $(\Delta)$ at the defined time points of the time-kill assay

\begin{tabular}{|c|c|c|c|c|c|c|c|}
\hline Antibiotic or antibiotic combination & $\begin{array}{l}\text { Day } 0 \\
\left(\log _{10} \mathrm{CFU} \mathrm{ml} \mathrm{m}^{-1}\right)\end{array}$ & $\begin{array}{l}\text { Day } 1 \\
\left(\log _{10} \mathrm{CFU} \mathrm{ml} \mathrm{m}^{-1}\right)\end{array}$ & $\Delta$ Day 1 & $\begin{array}{l}\text { Day } 3 \\
\left(\log _{10} \mathrm{CFU} \mathrm{ml} \mathrm{m}^{-1}\right)\end{array}$ & $\Delta$ Day 3 & $\begin{array}{l}\text { Day } 6 \\
\left(\log _{10} \mathrm{CFU} \mathrm{ml} \mathrm{m}^{-1}\right)\end{array}$ & $\Delta$ Day 6 \\
\hline GC & $6.85 \pm 0.09$ & $7.35 \pm 0.42$ & 0.5 & $8.18 \pm 0.39$ & 1.33 & $8.80 \pm 0.65$ & 1.95 \\
\hline AMK & $6.85 \pm 0.10$ & $6.37 \pm 0.38$ & -0.48 & $5.62 \pm 0.49$ & -1.23 & $4.84 \pm 0.92$ & -2.01 \\
\hline TGC & $6.85 \pm 0.11$ & $6.73 \pm 0.43$ & -0.12 & $6.50 \pm 0.33$ & -0.35 & $6.39 \pm 0.31$ & -0.46 \\
\hline IPM & $6.85 \pm 0.12$ & $6.12 \pm 0.64$ & -0.73 & $5.47 \pm 0.94$ & -1.38 & $5.37 \pm 0.35$ & -1.48 \\
\hline CLR & $6.85 \pm 0.13$ & $6.58 \pm 0.42$ & -0.27 & $6.02 \pm 0.40$ & -0.83 & $5.58 \pm 0.60$ & -1.27 \\
\hline AMK/TGC/IPM & $6.85 \pm 0.14$ & $6.10 \pm 0.75$ & -0.75 & $5.42 \pm 0.60$ & -1.43 & $4.27 \pm 0.67$ & -2.58 \\
\hline AMK/TGC/CLR & $6.85 \pm 0.15$ & $6.20 \pm 0.49$ & -0.65 & $5.46 \pm 0.70$ & -1.39 & $3.83 \pm 0.90$ & -3.02 \\
\hline
\end{tabular}

$C F U$ colony-forming unit, $G C$ growth control, $A M K$ amikacin, $T G C$ tigecycline, IPM imipenem, $C L R$ clarithromycin

differences $(P \geq 0.05)$ were observed between the activities of the two three-drug combinations.

\section{Discussion}

In this study, the effects of amikacin, tigecycline, imipenem, and clarithromycin individually and in two three-drug combinations were investigated as these antibiotics are usually administered during the treatment of lung infections caused by M. abscessus. To our knowledge, there are few studies on the effect of these antibiotics against M. abscessus clinical isolates using the time-kill assay [12, 13]. This method is adequate for studying the activity of antibiotics and determining their pharmacodynamics [14]. Furthermore, the time-kill assay has been largely used in clinical microbiology laboratories and has demonstrated to be reliable and consistent in the study of different microorganisms, including NTM [11, 15-18]. The antibiotic susceptibility testing for $M$. abscessus is read at day 3 in microbiological practice. However, in this study the time points were established at days 3 and 6 in order to study the accumulated activity of the antibiotics alone and of the three-drug combinations. Regarding the antibiotics, it is well-known that tigecycline has poor stability. In this study, it was prepared as described by Jitkova et al. and remained stable for up to 7 days [10]. This novel formulation allows the testing of tigecycline, a drug frequently used in the treatment of M. abscessus lung infections given that it is more effective than doxycycline and other tetracyclines [8]. Concerning imipenem, it shows poor stability in formulations parenterally administered to patients. This poor stability has been attributed to changes in $\mathrm{pH}$ and in the concentrations of sodium bisulfite and Lcysteine [19]. Nonetheless, our in vitro results showed activity of all the antibiotics and antibiotic combinations at both days 3 and 6.

Currently, the majority of patients with lung infections by $M$. abscessus receive more than two antibiotics in combination during the course of treatment. However, there is no standard combination and most of the treatments are based on empirical experience. In addition, most studies only investigate the activity of antibiotics in two-drug combinations. Some of them include few of the antibiotics analyzed in the present study, but none includes three-drug combinations $[12,15,16]$.

In this study we used isolates of M. abscessus subsp. abscessus, the most isolated subspecies in our area. From a strain collection we selected seven clinical isolates susceptible to macrolides, with the aim to compare the two three-drug combinations. The results show that both combinations had good activity and did not show statistically significant differences, although the reduction in $\log _{10} \mathrm{CFU} \mathrm{ml}{ }^{-1}$ with the combination including imipenem was lower than of that including clarithromycin. For this reason and due to the IV administration of imipenem, the combination of amikacin/tigecycline/imipenem could not be recommended as an alternative in the treatment of $M$. abscessus subsp. abscessus lung infections caused by clarithromycin susceptible isolates. However, in the light of the results obtained, this combination could be considered against clarithromycin resistant isolates. Future studies are required to confirm this hypothesis. This is especially important, since at least 14 months of treatment are necessary and outcomes remain very poor, with success rates of only $30-50 \%$ [20].

Furthermore, systems of antibiotic administration that have already proven to be effective against NTM lung infections, such as inhaled liposomal antibiotics and other nanoparticle-based antibiotic delivery systems, should be further developed. These forms of administration are currently used for amikacin [21]. In addition, imipenem physicochemical properties also allow its administration using these new delivery systems [22].

In conclusion, both three-drug combinations, amikacin/ tigecycline/imipenem, and amikacin/tigecycline/clarithromycin, showed similar in vitro activity against $M$. abscessus subsp. abscessus clinical isolates as well as no antagonistic activity. The combination including imipenem is not a reliable alternative against $M$. abscessus subsp. abscessus lung infections caused by isolates susceptible to clarithromycin. However, this combination should be further studied against clarithromycin resistant isolates. 
Acknowledgements The authors belong to the Study Group of Mycobacterial Infections (GEIM) of the Sociedad Española de Enfermedades Infecciosas y Microbiología Clínica (SEIMC), the Spanish Network for the Research in Infectious Diseases (REIPI) and the research team awarded for quality control by Agència de Gestió d'Ajuts Universitaris $i$ de Recerca [AGAUR, 2017SGR0809]. ISGlobal is a member of the CERCA Program, Generalitat de Catalunya. In addition, we are deeply grateful to Donna Pringle for her help with the English redaction.

Funding This work was supported by the Ministerio de Economía y Competitividad, Instituto de Salud Carlos III, co-financed by the European Regional Development Fund (ERDF) 'A way to achieve Europe', the Spanish Ministry of Health (grant no. PI16/01047), Planes Nacionales de I+D+i 2008-2011/2013-2016 and Instituto de Salud Carlos III, Subdirección General de Redes y Centros de Investigación Cooperativa, Ministerio de Economía y Competitividad, Spanish Network for Research in Infectious Diseases (REIPI) (RD16/ 0016/0010) co-financed by European Development Regional Fund (ERDF) "A way to achieve Europe" and operative program Intelligent Growth 2014-2020. This study was also supported by grant 2017SGR0809 from the Departament d'Universitats, Recerca $i$ Societat de la Informació de la Generalitat de Catalunya and by a grant from Fundació La Marató de TV3 (grant no. 201816-10). EP-B received a grant from the Universitat de Barcelona (UB), Ajut de Personal Investigador en Formació (APIF-UB).

\section{Compliance with ethical standards}

Conflict of interest The authors declare that they have no conflict of interest.

Ethical approval Ethical approval was received from the Ethical Committee of the Hospital Clínic de Barcelona (Barcelona, Spain) [HCB/2016/0344].

Publisher's note Springer Nature remains neutral with regard to jurisdictional claims in published maps and institutional affiliations.

Open Access This article is licensed under a Creative Commons Attribution 4.0 International License, which permits use, sharing, adaptation, distribution and reproduction in any medium or format, as long as you give appropriate credit to the original author(s) and the source, provide a link to the Creative Commons license, and indicate if changes were made. The images or other third party material in this article are included in the article's Creative Commons license, unless indicated otherwise in a credit line to the material. If material is not included in the article's Creative Commons license and your intended use is not permitted by statutory regulation or exceeds the permitted use, you will need to obtain permission directly from the copyright holder. To view a copy of this license, visit http://creativecommons. org/licenses/by/4.0/.

\section{References}

1. Parte AC. LPSN - List of Prokaryotic names with Standing in Nomenclature (bacterio.net), 20 years on. Int J Syst Evol Microbiol. 2018;68:1825-9.

2. Lopeman RC, Harrison J, Desai M, Cox JAG. Mycobacterium abscessus: environmental bacterium turned clinical nightmare. Microorganisms. 2019;7:90.
3. Gupta RS, Lo B, Son J. Phylogenomics and comparative genomic studies robustly support division of the genus Mycobacterium into an emended genus Mycobacterium and four novel genera. Front Microbiol. 2018;9:67.

4. Zhang Z, Lu J, Song Y, Pang Y. In vitro activity between linezolid and other antimicrobial agents against Mycobacterium abscessus complex. Diagn Microbiol Infect Dis. 2018;90:31-4.

5. Davidson RM. A closer look at the genomic variation of geographically diverse Mycobacterium abscessus clones that cause human infection and disease. Front Microbiol. 2018;9:2988.

6. Aono A, Morimoto K, Chikamatsu K, Yamada H, Igarashi Y, Murase Y, et al. Antimicrobial susceptibility testing of Mycobacteroides (Mycobacterium) abscessus complex, Mycolicibacterium (Mycobacterium) fortuitum, and Mycobacteroides (Mycobacterium) chelonae. J Infect Chemother. 2019;25:117-23.

7. Haworth CS, Banks J, Capstick T, Fisher AJ, Gorsuch T, Laurenson IF, et al. British Thoracic Society guidelines for the management of non-tuberculous mycobacterial pulmonary disease (NTM-PD). Thorax. 2017;72:ii1-ii64. https://doi.org/10.1136/ thoraxjnl-2017-210927.

8. Wallace RJ Jr, Brown-Elliott BA, Crist CJ, Mann L, Wilson RW. Comparison of the in vitro activity of the glycylcycline tigecycline (formerly GAR-936) with those of tetracycline, minocycline, and doxycycline against isolates of nontuberculous mycobacteria. Antimicrob Agents Chemother. 2002;46:3164-7.

9. Ferro BE, van Ingen J, Wattenberg M, van Soolingen D, Mouton JW. Time-kill kinetics of antibiotics active against rapidly growing mycobacteria. J Antimicrob Chemother. 2015;70:811-7.

10. Jitkova Y, Gronda M, Hurren R, Wang X, Goard CA, Jhas B, et al. A novel formulation of tigecycline has enhanced stability and sustained antibacterial and antileukemic activity. PLoS ONE. 2014;9:e95281 https://doi.org/10.1371/journal.pone.0095281.

11. López-Gavín A, Tudó G, Rey-Jurado E, Vergara A, Hurtado JC, Gonzalez-Martín J. In vitro time-kill curves study of three combinations against Mycobacterium tuberculosis clinical isolates. Int J Antimicrob Agents. 2016;47:97-100.

12. Ferro BE, Meletiadis J, Wattenberg M, de Jong A, van Soolingen D, Mouton JW, et al. Clofazimine prevents the regrowth of Mycobacterium abscessus and Mycobacterium avium type strains exposed to amikacin and clarithromycin. Antimicrob Agents Chemother. 2016;60:1097-105.

13. Lefebvre AL, Dubée V, Cortes M, Dorchêne D, Arthur M, Mainardi JL. Bactericidal and intracellular activity of $\beta$-lactams against $\mathrm{Myco}$ bacterium abscessus. J Antimicrob Chemother. 2016;71:1556-63.

14. Lorian V. Antibiotics in laboratory medicine. 5th ed. Philadelphia: Williams \& Wilkins; 2005.

15. Pandey R, Chen L, Manca C, Jenkins S, Glaser L, Vinnard C, et al. Dual $\beta$-lactam combinations highly active against Mycobacterium abscessus complex in vitro. MBio. 2019;10:1.

16. Pryjma M, Burian J, Thompson CJ. Rifabutin acts in synergy and is bactericidal with frontline Mycobacterium abscessus antibiotics clarithromycin and tigecycline, suggesting a potent treatment combination. Antimicrob Agents Chemother. 2018;62:e00283-18. https://doi.org/10.1128/AAC.00283-18.

17. Rey-Jurado E, Tudó G, Soy D, González-Martín J. Activity and interactions of levofloxacin, linezolid, ethambutol and amikacin in three-drug combinations against Mycobacterium tuberculosis isolates in a human macrophage model. Int J Antimicrob Agents. 2013;42:524-30.

18. Bax HI, Bakker-Woudenberg IAJM, Kate MTT, Verbon A, De Steenwinkelb JEM. Tigecycline potentiates clarithromycin activity against Mycobacterium avium in vitro. Antimicrobial Antimicrob Agents Chemother. 2016;60:2577-9.

19. Yoshida M, Takasu Y, Shimizu K, Asahara K, Uchida T. Prediction of the stability of imipenem in intravenous mixtures. Chem Pharm Bull. 2013;61:1-7. 
20. Nathavitharana RR, Strnad L, Lederer PA, Shah M, Hurtado RM. Top questions in the diagnosis and treatment of pulmonary $M$. abscessus disease. Open Forum Infect Dis. 2019;6:ofz221 https://doi.org/10.1093/ofid/ofz221.

21. Rose SJ, Neville ME, Gupta R, Bermudez LE. Delivery of aerosolized liposomal amikacin as a novel approach for the treatment of nontuberculous mycobacteria in an experimental model of pulmonary infection. PLoS ONE. 2014;9:e108703 https://doi.org/ 10.1371/journal.pone.0108703.

22. Todisco T, Eslami A, Baglioni S, Sposini T, Tascini C, Sommer E, et al. Basis for nebulized antibiotics: droplet characterization and in vitro antimicrobial activity versus Staphylococcus aureus, Escherichia coli, and Pseudomonas aeruginosa. J Aerosol Med. 2000;13:11-6. 